\title{
Correlation between atrial fibrillation, serum amyloid protein $A$ and other inflammatory cytokines
}

\author{
TAO CHENG $^{1}$, XIAO-FEI WANG ${ }^{2}$, YUN-TIAN HOU ${ }^{3}$ and LI ZHANG ${ }^{4}$ \\ ${ }^{1}$ Beijing Institute of Transfusion Medicine; ${ }^{2}$ Beijing Institute of Pharmacology and Toxicology; ${ }^{3} 301$ st Hospital of Beijing, \\ Beijing 100850; ${ }^{4}$ Shandong University, Jinan 250012, P.R. China
}

Received January 13, 2012; Accepted April 24, 2012

DOI: $10.3892 / \mathrm{mmr} .2012 .934$

\begin{abstract}
Atrial fibrillation (AF) may occur and persist due to atrial remodeling exacerbated by inflammation. However, whether inflammatory cytokines, such as serum amyloid protein A (SAA), are elevated in patients with atrial arrhythmias remains unclear. In a case-control study design, serum levels of inflammatory cytokines SAA, IL-1, IL-6, TNF- $\alpha$ and high-sensitivity C-reactive protein (hsCRP) were compared between 122 patients with AF and 63 non-AF controls. Of 122 AF patients, 48 had lone AF and 74 organic AF; 65 had paroxysmal AF, 36 persistent $\mathrm{AF}$ and 21 permanent $\mathrm{AF}$. In unadjusted analyses, the levels of SAA, hsCRP, TNF- $\alpha$ P, IL-1 and IL-6 were significantly higher in AF patients compared to control patients $(\mathrm{P}<0.01)$. Patients with permanent and persistent AF had higher serum levels of inflammatory cytokines than those with paroxysmal AF; all AF patients had higher serum levels than controls $(\mathrm{P}<0.05)$. Using a multivariate logistic regression analysis, we found that SAA (OR 3.11; 95\% CI, 1.12-6.05), hsCRP (OR 2.53; 95\% CI, 1.17-4.38), TNF- $\alpha$ (OR 1.80 ; 95\% CI, 1.03-3.20) and IL-6 (OR 1.21; 95\% CI, 0.68-2.87) were correlated with AF $(\mathrm{P}<0.05)$. SAA and related inflammatory cytokines are elevated in AF patients. This study is the first to document elevated SAA in AF patients. Although the cause of elevated SAA levels in AF patients remains unknown, elevated SAA may reflect an inflammatory state that promotes the occurrence and persistence of AF.
\end{abstract}

\section{Introduction}

Atrial fibrillation (AF) is the most common arrhythmia; at least 2.3 million individuals suffer from AF in the US alone (1). The etiology of AF is not completely understood. Many factors such as neuro-endocrine function, acute or chronic hemodynamics and metabolism may cause atrial remodeling and thus play a role in the initiation and progression of AF.

Correspondence to: Dr Tao Cheng, Beijing Institute of Transfusion Medicine, No. 27 Taiping Rd, Haidian District, Beijing 100850, P.R. China

E-mail: chentao100850@126.com

Key words: atrial fibrillation, serum amyloid protein A, inflammation
Previous studies showed a close correlation between inflammation and AF (2). In 1997, two groups reported inflammatory changes in the atrial tissue of AF patients $(3,4)$. Frustaci et al biopsied atrial myocardium from the right atrial septum of 12 paroxysmal and lone AF patients and found inflammatory changes in the atrial tissue of each biopsy, while all 11 Wolf-Parkinson-White syndrome (WPW) patients in the control group had normal atrial biopsy (3). Conventional anti-inflammatory agents such as glucocorticoid and new options such as statins are able to abate myocardial inflammation, lower the C-reactive protein (CRP) level, which is elevated in inflammation, reduce AF relapse, and decrease the duration of AF. The anti-inflammatory effect appears to be more significant in cases of higher CRP levels. Therefore, rather than being a result of $\mathrm{AF}$, the inflammatory changes in atrial tissue may be the driving force of $\mathrm{AF}(5,6)$.

Inflammation of atrial tissue leads to the denaturation, necrosis, apoptosis, fibrosis and scarring of myocardial cells. Such changes alter their electrophysiological function, increase their non-uniform and anisotropic properties, and decrease the conduction of atrial muscles, facilitating reentry of the electrical signal and causing AF to persist. Interstitial fibrosis of atrial cells may be involved in atrial structural remodeling (7). Levels of two inflammatory markers, CRP and interleukin (IL)-6, are positively correlated with left atrial diameter (8); thus, inflammation may play a role in atrial remodeling and prolong the duration of AF.

An acute-phase inflammatory protein similar to CRP, serum amyloid A (SAA) protein, appears to be more sensitive to inflammatory stimulation than CRP (9). SAAs are a family of conserved proteins encoded by one gene cluster. While synthesized predominantly in liver cells, epithelial cells in certain human tissues are also capable of expressing some types of SAA (10). SAA mRNA is expressed in the majority of endothelial cells within human coronary arteries and AS lesions, some smooth muscle cells and macrophage-derived foam cells, adventitial macrophages, vascular interstitial cells and even fat cells (11). Upon entering the plasma, SAA attaches to high density lipoprotein (HDL). Unlike CRP, SAA mostly binds to HDL-C when it exceeds the normal level, which makes its behavior similar to apolipoprotein. By stimulating the secretion of matrix metalloprotease in macrophages and collagenase in smooth muscle cells, SAAs may degrade collagen, extracellular matrix and stromatin, and 
thus facilitate tissue remodeling. SAA is also an ECM binding protein inducing platelet aggregation and production of matrix metalloproteases. Increase in matrix metalloproteases in the atrium often coincides with increased apoptosis, cell death, inflammatory infiltration and evident fibrosis. Additionally, according to a recent study (12), SAA is highly correlated with atherosclerosis and coronary heart disease. Despite these indications of a role for SAA in atrial remodeling, an association of SAAs with AF has not yet been reported.

In response to inflammation, the amount of SAA in plasma, which is normally minimal, becomes elevated by $4-6 \mathrm{~h}$ after stimulation of inflammation or other injuries (trauma, infection and tumors) and peaks at 8-12 $\mathrm{h}$ at levels up to 1,000 times higher than normal (11). Thus, SAA is a sensitive marker of inflammation. Patients with acute and chronic inflammation usually have higher SAA levels, and long-term and repeated infection causes persistent elevation in SAA.

To obtain more data regarding the correlation between inflammation and AF, we investigated specific inflammatory cytokines such as SAA and interleukins in patients with AF.

\section{Materials and methods}

Study population. The study population included 122 patients with AF treated at the Beijing 301st Hospital between March 2005 and January 2011. For controls, 63 patients with sinus rhythm and no history of AF, as confirmed in a routine physical examination during the same period, were recruited. AF patients were assigned to groups as follows: by etiology, 48 had lone atrial fibrillation and 74 had organic lesion; by duration of AF, 65 had paroxysmal AF, 36 had persistent $\mathrm{AF}$ and 21 had permanent atrial fibrillation. Patients in the AF and control groups were matched for basic factors affecting SAA, including gender and age (Table I). Patients provided written informed consent, and ethics approval was obtained from the Institute of Jing Feng Medical Laboratory.

Patients were thoroughly examined to determine general and cardiac health. Echocardiogram (ECG) was used to evaluate myocardial ischemia, ejection fraction and cardiac structural change (atrial enlargement or left ventricular hypertrophy). Other clinical data, including body mass index, blood pressure, blood lipids and fasting blood sugar were collected as normal.

Criteria for exclusion included presence of communicable diseases, injuries within 1 month or surgery within 60 days prior to the study, acute coronary syndrome in the month of study, chronic inflammatory disease, tuberculosis, malignancy or immunological disease, hepatic and renal insufficiency, ejection fraction $<0.6$ and evidence of clinical heart failure or valvular heart disease.

Case definitions. Lone atrial fibrillation (LAF) was defined as AF occurring in patients without structural heart disease, including patients with hypertension. Based on the duration of disease, $\mathrm{AF}$ was categorized as paroxysmal ( $<30$ days), persistent ( $\geq 30$ days and $<1$ year) and permanent $(\geq 1$ year). Patients with paroxysmal AF had blood collected for cytokine assessment when the sinus heart rate was measured.

Blood collection. To measure serum inflammatory cytokines, $5 \mathrm{ml}$ venous blood was obtained from fasting participants in
Table I. Baseline patient characteristics (mean $\pm \mathrm{SD}$ ).

\begin{tabular}{lccc}
\hline & Control $(\mathrm{n}=63)$ & AF $(\mathrm{n}=122)$ & P-value \\
\hline Age, years & $52.4 \pm 0.8$ & $60.7 \pm 2.3$ & $<0.05$ \\
Male gender, n $(\%)$ & $39(61.9)$ & $48(37.7)$ & $<0.01$ \\
BMI $\left(\mathrm{kg} / \mathrm{m}^{2}\right)$ & $23.1 \pm 2.0$ & $28.3 \pm 2.9$ & $<0.05$ \\
TG $(\mathrm{mmol} / \mathrm{l})$ & $1.40 \pm 0.28$ & $1.94 \pm 0.55$ & $<0.05$ \\
TC $(\mathrm{mmol} / \mathrm{l})$ & $4.18 \pm 1.03$ & $5.46 \pm 1.44$ & $<0.01$ \\
LDL $(\mathrm{mmol} / \mathrm{l})$ & $3.06 \pm 1.21$ & $4.22 \pm 1.29$ & $<0.01$ \\
HDL $(\mathrm{mmol} / \mathrm{l})$ & $1.27 \pm 0.23$ & $0.76 \pm 0.15$ & $<0.05$ \\
FBG $(\mathrm{mmol} / \mathrm{l})$ & $5.60 \pm 1.05$ & $6.94 \pm 1.88$ & $<0.01$ \\
Smoking, $(\%)$ & $24(38.1)$ & $51(41.8)$ & $>0.05$ \\
Hypertension, $\mathrm{n}(\%)$ & $15(23.8)$ & $65(53.3)$ & $<0.01$ \\
CAD, $\mathrm{n}(\%)$ & $0(0)$ & $28(22.9)$ & $<0.01$ \\
DCM, n $(\%)$ & $0(0)$ & $14(11.5)$ & $<0.05$ \\
HCM, n $(\%)$ & $0(0)$ & $2(1.6)$ & $>0.05$ \\
TIA $/$ CVA, n $(\%)$ & $0(0)$ & $21(17.2)$ & $<0.01$ \\
\hline
\end{tabular}

Values are the mean \pm SD or n (\%). BMI, body mass index; TG, triglyceride; TC, total cholesterol; LDL, low-density lipoprotein; HDL, high density lipoprotein; FBG, fasting blood glucose; CAD, coronary artery disease; DCM, dilated cardiomyopathy; HCM, hypertrophic cardiomyopathy; TIA/CVA, history of transient ischemic attack or cerebrovascular accident.

the morning, treated in an anticoagulant tube and centrifuged at $1000 \mathrm{rpm}$ for $10 \mathrm{~min}$; plasma was stored at $-80^{\circ} \mathrm{C}$ until testing.

Serum cytokine measurements. Enzyme-linked immunosorbent assay (ELISA) was used to measure serum cytokines via the two-antibody sandwich method (13). Briefly, primary antibody was coated onto 96-well Co-Star plates (Cole-Parmer, Vernon Hills, IL, USA) to which were added serum samples containing the antigen to be examined. After thorough washing, secondary antibody conjugated to horseradish peroxidase was applied for detection. Color was developed with o-phenylenediamine (OPD; Sigma Chemical Company, St. Louis, MO, USA) prior to visualization with the ELISA reader. Color intensity was proportional to the concentration of the antigen. A linear gradient standard curve was plotted, and the unknown was determined from this standard curve. The SAA (Market, USA), IL- 6 and TNF- $\alpha$ (Genzyme, Cambridge, MA, USA), and hsCRP (Diagnostic System Laboratory, Franklin Lakes, NJ, USA) were measured using kits according to the manufacturer's instructions. An automated microplate reader (ELX800 Microplate Reader, Cary, NC, USA) was used to measure color intensity at $450 \mathrm{~nm}$.

Statistical analysis. With a positively skewed distribution, SAA was described by median and interquartile range (IQR), and differences between groups were compared using the Wilcoxon rank-sum test. Other measurement data showing normal distribution were summarized using the means and standard deviation. Two sample t-tests, analysis of variance (ANOVA) and Chi-square tests were used for unadjusted comparisons between groups when applicable. A multivariate 
Table II. Serum levels of inflammatory cytokines in the AF and control groups.

\begin{tabular}{|c|c|c|c|c|c|c|}
\hline Group & $\mathrm{n}$ & $\begin{array}{c}\text { SAA median (IQR) } \\
\mathrm{mg} / 1\end{array}$ & $\begin{array}{l}\text { hsCRP median (IQR) } \\
\text { mg/l }\end{array}$ & $\begin{array}{c}\text { TNF- } \alpha \\
\text { ng/l }\end{array}$ & $\begin{array}{l}\mathrm{IL}-1 \\
\mathrm{ng} / 1\end{array}$ & $\begin{array}{l}\mathrm{IL}-6 \\
\mathrm{ng} / 1\end{array}$ \\
\hline Control & 63 & $1.27(1.05-1.92)$ & $0.79(0.38-2.70)$ & $5.94 \pm 2.34$ & $4.43 \pm 1.62$ & $2.90 \pm 1.02$ \\
\hline $\mathrm{AF}$ & 122 & $3.43(2.01-5.58)^{\mathrm{b}}$ & $2.35(1.07-5.25)^{\mathrm{b}}$ & $10.88 \pm 3.56^{\mathrm{b}}$ & $8.06 \pm 2.75^{b}$ & $6.39 \pm 2.10^{\mathrm{b}}$ \\
\hline Lone AF & 48 & $2.54(1.31-4.71)^{\mathrm{a}}$ & $1.45(0.49-5.40)^{\mathrm{a}}$ & $7.35 \pm 2.09^{\mathrm{a}}$ & $6.10 \pm 1.79^{a}$ & $4.50 \pm 0.95^{\mathrm{a}}$ \\
\hline Organic AF & 74 & $3.84(2.07-7.54)^{\mathrm{a}, \mathrm{c}}$ & $2.81(1.21-6.39)^{\mathrm{a}, \mathrm{c}}$ & $11.60 \pm 3.88^{\mathrm{a}, \mathrm{c}}$ & $8.94 \pm 2.55^{\mathrm{a}, \mathrm{c}}$ & $7.06 \pm 2.03^{\mathrm{a}, \mathrm{c}}$ \\
\hline Paroxysmal AF & 65 & $2.66(1.40-4.88)^{\mathrm{a}}$ & $1.90(0.57-4.14)^{\mathrm{a}}$ & $7.05 \pm 1.95^{\mathrm{a}}$ & $6.85 \pm 1.47^{\mathrm{a}}$ & $4.61 \pm 1.17^{\mathrm{a}}$ \\
\hline Persistent AF & 36 & $4.03(2.97-9.53)^{\mathrm{a}, \mathrm{d}}$ & $2.53(1.08-6.95)^{\mathrm{a}, \mathrm{d}}$ & $11.04 \pm 3.03^{\mathrm{a}, \mathrm{d}}$ & $9.10 \pm 2.03^{\mathrm{a}, \mathrm{d}}$ & $6.56 \pm 1.09^{\mathrm{a}, \mathrm{d}}$ \\
\hline Permanent AF & 21 & $3.95(2.76-9.10)^{\mathrm{a}, \mathrm{d}}$ & $2.03(0.89-5.90)^{\mathrm{a}, \mathrm{d}}$ & $9.37 \pm 2.85^{\mathrm{a}, \mathrm{d}}$ & $8.58 \pm 2.395^{\mathrm{a}, \mathrm{d}}$ & $5.50 \pm 1.25^{\mathrm{a}, \mathrm{d}}$ \\
\hline
\end{tabular}

vs. Control, ${ }^{\mathrm{a}} \mathrm{P}<0.05$, ${ }^{\mathrm{b}} \mathrm{P}<0.01$; vs. lone $\mathrm{AF},{ }^{\mathrm{c}} \mathrm{P}<0.05$; vs. paroxysmal $\mathrm{AF},{ }^{\mathrm{d}} \mathrm{P}<0.05$. $\mathrm{AF}$, atrial fibrillation; $\mathrm{SAA}$, serum amyloid protein $\mathrm{A} ; \mathrm{IQR}$, interquartile range.

logistic regression model was developed to investigate the association between multiple inflammatory cytokines and AF after controlling for age, gender and BMI. Statistical analyses were conducted using the SPSS 12.0 statistical package. $\mathrm{P}<0.05$ was considered to indicate a statistically significant difference.

\section{Results}

Baseline characteristics of patients with AF. Patients with AF were more likely to be female and older (Table I). Additionally, this group exhibited higher body weights, triglycerides (TG), total cholesterol (TC), low density lipoprotein (LDL) and fasting blood glucose (FBG), with lower HDL compared to controls. Moreover, patients with AF were more likely to have a history of hypertension, coronary disease, dilated myocardiopathy, transient ischemic attack (TIA) or cerebrovascular accident (CVA) compared to patients in the control group. All of these factors were statistically significantly different between groups. However, smoking and hypertrophic cardiomyopathy (HCM) were not significantly different between groups.

Comparison of inflammatory cytokines among various AF subgroups. Using ELISA, serum levels of the inflammatory cytokines SAA, IL-1, IL-6, TNF- $\alpha$ and hsCRP in patients with AF and the control group were measured (Table II). As a group, patients with AF exhibited elevated levels of each of these cytokines compared to controls. For AF subgroups, serum levels of each cytokine were also higher than in the control group. Additionally, cytokines in the organic AF subgroup were higher than in the LAF subgroup and, similarly, both persistent and permanent AF subgroups exhibited higher levels than the paroxysmal AF subgroup. However, no significant differences in the serum levels of SAA, IL-1, IL-6, TNF- $\alpha$ or hsCRP were found between persistent AF patients and paroxysmal AF patients.

Correlation between inflammatory cytokines and $A F$. Univariate logistic regression analysis was used to identify any potential correlation between elevated serum cytokine levels and the presence of AF (Table III). A positive correlation with AF was found for not only the serum cytokines SAA,
Table III. Correlation of SAA and other inflammatory cytokines with AF by logistic regression.

Odds ratio $(95 \% \mathrm{CI})$ for atrial fibrillation

Multivariate
(adjusted)

\begin{tabular}{lll}
\hline SAA & $2.83(0.90-5.29)^{\mathrm{a}}$ & $3.11(1.12-6.05)^{\mathrm{a}}$ \\
hsCRP & $3.06(1.63-4.54)^{\mathrm{a}}$ & $2.53(1.17-4.38)^{\mathrm{a}}$ \\
TNF- $\alpha$ & $1.94(1.12-3.05)^{\mathrm{a}}$ & $1.80(1.03-3.20)^{\mathrm{a}}$ \\
IL-1 & $1.30(0.85-2.61)^{\mathrm{a}}$ & $1.15(0.63-2.23)$ \\
IL-6 & $1.55(0.71-2.79)^{\mathrm{a}}$ & $1.21(0.68-2.87)^{\mathrm{a}}$ \\
Age, y & $2.73(1.68-4.18)^{\mathrm{a}}$ & $2.87(1.77-4.36)^{\mathrm{a}}$ \\
Male gender, n $(\%)$ & $0.61(0.45-2.19)^{\mathrm{a}}$ & $0.59(0.34-2.06)^{\mathrm{a}}$ \\
BMI $\left(\mathrm{kg} / \mathrm{m}^{2}\right)$ & $2.01(0.87-3.13)^{\mathrm{a}}$ & $1.98(0.75-2.96)^{\mathrm{a}}$ \\
TG $(\mathrm{mmol} / \mathrm{l})$ & $1.18(0.74-2.80)$ & $1.04(0.60-2.47)$ \\
TC $(\mathrm{mmol} / \mathrm{l})$ & $1.65(1.05-2.63)^{\mathrm{a}}$ & $1.60(0.98-2.55)^{\mathrm{a}}$ \\
LDL $(\mathrm{mmol} / \mathrm{l})$ & $1.81(0.95-3.08)^{\mathrm{a}}$ & $1.15(0.96-2.90)$ \\
HDL $(\mathrm{mmol} / \mathrm{l})$ & $0.92(0.30-3.24)^{\mathrm{a}}$ & $0.85(0.72-2.96)$ \\
FBG $(\mathrm{mmol} / \mathrm{l})$ & $2.86(0.75-4.78)^{\mathrm{a}}$ & $3.01(0.88-5.34)^{\mathrm{a}}$ \\
Smoking, n $(\%)$ & $1.24(0.63-2.84)^{\mathrm{a}}$ & $1.12(0.52-2.80)$ \\
Hypertension, n $(\%)$ & $2.58(1.05-3.69)^{\mathrm{a}}$ & $1.91(0.88-3.15)^{\mathrm{a}}$ \\
CAD, n $(\%)$ & $4.23(1.79-10.37)^{\mathrm{a}}$ & $2.31(1.02-4.14)^{\mathrm{a}}$ \\
DCM, n $(\%)$ & $4.94(1.52-11.71)^{\mathrm{a}}$ & $2.89(1.16-3.85)^{\mathrm{a}}$ \\
HCM, n $(\%)$ & $1.13(0.86-2.52)$ & $1.14(0.87-2.61)$ \\
TIA/CVA, n $(\%)$ & $3.06(1.31-7.48)^{\mathrm{a}}$ & $2.28(0.93-3.87)^{\mathrm{a}}$ \\
\hline
\end{tabular}

${ }^{\mathrm{a}} \mathrm{P}<0.05$. SAA, serum amyloid protein $\mathrm{A} ; \mathrm{AF}$, atrial fibrillation; hsCRP, high-sensitivity C-reactive protein; BMI, body mass index; TG, triglycerides; TC, total cholesterol; LDL, low-density lipoprotein; HDL, high-density lipoprotein; FBG, fasting blood glucose; CAD, coronary artery disease; DCM, dilated cardiomyopathy; HCM, hypertrophic cardiomyopathy; TIA/CVA, history of transient ischemic attack or cerebrovascular accident.

hsCRP, TNF- $\alpha$ and IL-6, but also for age, BMI, TC, LDL, FBG, smoking, hypertension, coronary artery disease (CAD), dilated cardiomyopathy (DCM) and TIA/CVA $(<5 \%)$. A negative correlation was found between $\mathrm{AF}$ and both male gender 
and HDL $(<5 \%)$. However, no clear correlation was found between IL-1, TG, HCM and AF ( $>5 \%)$. A multivariate logistic regression analysis indicated that SAA, hsCRP, TNF- $\alpha$, IL-6, age, BMI, TC, LDL, FBG, hypertension, CAD, DCM and TIA/CVA were positively correlated with $\mathrm{AF}(<5 \%)$, but male gender was negatively correlated with AF $(<5 \%)$. IL-1, TG, LDL, HDL, HCM and smoking were not significantly correlated with $\mathrm{AF}(>5 \%)$.

\section{Discussion}

Isolated atrial amyloidosis (IAA) is the most common manifestation of senile amyloidosis. Evidence indicates that IAA plays an important role in the etiology of AF. The incidence and severity of IAA increase with age, occurring in $75 \%$ of individuals aged 51-60 years (14). As SAA is involved in amyloidosis, research is required to determine whether IAA, and therefore $\mathrm{AF}$, is associated with the expression of SAA or other inflammatory cytokines. In this study, we demonstrate for the first time that SAA is elevated in patients with AF when compared to individuals without AF. Additionally, SAA in patients with both permanent and persistent $\mathrm{AF}$ is significantly higher than in patients with paroxysmal AF. Consistent with previous findings (15), serum CRP was also increased in LAF patients without structural heart disease. Therefore, we speculate that inflammation plays a role in both the initiation and maintenance of AF.

SAA was reported to increase with increasing FBG and the presence of insulin resistance (16). In the condition of obesity, fat cells (adipocytes) undergoing loss of normal function secrete inflammatory cytokines such as TNF- $\alpha$ and IL-6. Obesity is closely correlated with systemic inflammation and is more prominent in females (17). CRP is commonly used to measure the risk of CAD, a risk that is tightly correlated with obesity, but adipocytes express little CRP. Thus, SAA may be a better indicator for the risk of cardiovascular disease due to its increased expression in obese individuals. Obese fat tissue is 'inflammatory' as it decreases the expression of certain anti-inflammatory agents while increasing the expression of pro-inflammatory and thrombotic agents such as IL-6 and TNF- $\alpha$ (18). In this study, AF is shown to be positively correlated with patient age (OR 2.87; 95\% CI, 1.77-4.36), BMI (OR 1.98; 95\% CI, 0.75-2.96), TC (OR 1.60; 95\% CI, 0.98-2.55) and FBG (OR 3.01; 95\% CI, 0.88-5.34), underscoring the correlation between inflammation and conditions associated with obesity.

In conclusion, cytokines such as SAA, hsCRP, IL-6 and TNF- $\alpha$ are associated with the initiation and progression of AF. SAA may be an important inflammatory cytokine in predicting and possibly iducing AF. Results from this study may provide a basis for the prevention of AF. Nevertheless, the exact relationship between SAA and AF remains to be demonstrated.

\section{References}

1. Go AS, Hylek EM, Phillips KA, et al: Prevalence of diagnosed atrial fibrillation in adults: national implications for rhythm management and stroke prevention: the AnTicoagulation and Risk Factors in Atrial Fibrillation (ATRIA) Study. JAMA 285: 2370-2375, 2001.

2. Aviles RJ, Martin DO, Apperson-Hansen C, et al: Inflammation as a risk factor for atrial fibrillation. Circulation 108: 3006-3010, 2003.

3. Frustaci A, Chimenti C, Bellocci F, Morgante E, Russo MA and Maseri A: Histological substrate of atrial biopsies in patients with lone atrial fibrillation. Circulation 96: 1180-1184, 1997.

4. Bruins P, te Velthuis H, Yazdanbakhsh AP, et al: Activation of the complement system during and after cardiopulmonary bypass surgery: postsurgery activation involves $\mathrm{C}$-reactive protein and is associated with postoperative arrhythmia. Circulation 96: 3542-3548, 1997.

5. Kumagai K, Nakashima $\mathrm{H}$ and Saku K: The HMG-CoA reductase inhibitor atorvastatin prevents atrial fibrillation by inhibiting inflammation in a canine sterile pericarditis model. Cardiovasc Res 62: 105-111, 2004.

6. Dernellis J and Panaretou M: Relationship between C-reactive protein concentrations during glucocorticoid therapy and recurrent atrial fibrillation. Eur Heart J 25: 1100-1107, 2004.

7. Li D, Shinagawa K, Pang L, et al: Effects of angiotensinconverting enzyme inhibition on the development of the atrial fibrillation substrate in dogs with ventricular tachypacinginduced congestive heart failure. Circulation 104: 2608-2614, 2001.

8. Psychari SN, Apostolou TS, Sinos L, et al: Relation of elevated C-reactive protein and interleukin-6 levels to left atrial size and duration of episodes in patients with atrial fibrillation. Am J Cardiol 95: 764-767, 2005.

9. Gabay C and Kushner I: Acute-phase proteins and other systemic responses to inflammation. N Engl J Med 340: 448-454, 1999.

10. Urieli-Shoval S, Linke RP and Matzner Y: Expression and function of serum amyloid A, a major acute-phase protein, in normal and disease states. Curr Opin Hematol 7: 64-69, 2000.

11. Yang RZ, Lee MJ, Hu H, et al: Acute-phase serum amyloid A: an inflammatory adipokine and potential link between obesity and its metabolic complications. PLoS Med 3: 287, 2006.

12. King VL, Thompson J and Tannock LR: Serum amyloid A in atherosclerosis. Curr Opin Lipidol 22: 302-307, 2011.

13. Leng SX, McElhaney JE, Walston JD, Xie D, Fedarko NS and Kuchel GA: ELISA and multiplex technologies for cytokine measurement in inflammation and aging research. J Gerontol A Biol Sci Med Sci 63: 879-884, 2008.

14. Steiner I and Hajkova P: Patterns of isolated atrial amyloid: a study of 100 hearts on autopsy. Cardiovasc Pathol 15: 287-290, 2006.

15. Chung MK, Martin DO, Spricher D, et al: C-reactive protein elevation in patients with atrial arrhythmias: inflammatory mechanisms and persistence of atrial fibrillation. Circulation 104: 2886-2891, 2001.

16. Tannock LR, O'Brien KD, Knopp RH, et al: Cholesterol feeding increases $\mathrm{C}$-reactive protein and serum amyloid A levels in lean insulin-sensitive subjects. Circulation 111: 3058-3062, 2005.

17. Sze L, Schmid C, Bloch KE, Bernays R and Brändle M: Effect of transsphenoidal surgery on sleep apnoea in acromegaly. Eur $\mathrm{J}$ Endocrinol 156: 321-329, 2007.

18. Xu H, Barnes GT, Yang Q, et al: Chronic inflammation in fat plays a crucial role in the development of obesity related insulin resistance. J Clin Invest 112: 1821-1830, 2003. 